\section{Broken metallic tracheostomy tube migrating into the tracheobronchial tree}

\author{
Nilam U. Sathe, Ratna Priya, \\ Sheetal Shelke, Kartik Krishnan \\ Department of Ear, Nose and Throat, \\ King Edward Memorial Hospital and Seth \\ G.S. Medical College, Mumbai, India
}

\section{Abstract}

Foreign body aspiration can be a life-threatening emergency. Broken tracheostomy tube in tracheobronchial tree is one of the rarest types of foreign body reported. Here we report two cases of fracture of metallic tracheostomy tube, leading to foreign body in tracheobronchial tree. A 14-year-old girl presented to our Emergency Department with history of respiratory distress and violent bouts of cough since 2 days. Chest X-ray showed that the broken part of the tube was lodged in the right main bronchus. The presence of Parkinson's disease in the patient and restricted neck flexion offered a challenge both for the anaesthetist and the surgeon. We were successful in removing the broken tube in 13 small pieces. Check bronchoscopy was clear and the procedure went uneventful. We would like to conclude that broken tracheostomy tube presenting as foreign body bronchus is infrequent but it is a preventable complication of tarcheostomy. The patient must be kept on regular follow up to check for signs of wear and tear. Timely and periodic replacement of tracehostomy tube should also be done, otherwise such life-saving surgery can become lifethreatening.

\section{Introduction}

Foreign body aspiration can be a life-threatening emergency. Broken tracheostomy tube in the tracheobronchial tree is one of the rarest types of foreign body reported. Tracheostomy is a universal life-saving procedure for securing the airway and a lot of patients have to wear tracheostomy tube over a long period. Although the procedure is safe with minimal complications, fracture and hence aspiration of the tracheostomy tube can be potentially life-threatening. This can simply be avoided by ensuring proper followup visits. Here we report two cases of fracture of metallic tracheostomy tube, leading to foreign body in the tracheobronchial tree.

\section{Case \#1}

A 14-year-old girl presented to our Emergency Department with history of respiratory distress and violent bouts of cough since 2 days. There was a past history of Parkinson's disease and quadriplegia due to cervical spine fracture and the patient was tracheostomised following prolonged intubation for the same 5 years back. On examination she was afebrile, tachypnoeic with chest auscultation suggestive of reduced air entry on the right side. Further examination revealed that although the outer part of the metallic trachesostomy tube was in situ, the inner tube was missing below the collar. Chest X-ray showed that the broken part of the tube was lodged in the right main bronchus (Figure 1). The presence of Parkinson's disease in the patient and restricted neck flexion offered a challenge both for the anaesthetist and the surgeon. The patient was taken up for rigid bronchoscopy and a 6-mm scope was negotiated through the tracheostoma. Scopy confirmed the presence of a corroded part of the broken metallic tube in right main bronchus. The tube was so fragile that it was disintegrated into multiple small pieces and if we had not been careful there would have been high chances of displacement of those particles on attempt of removal. We removed the outer metallic tube first. Then, through the tracheostoma, we negotiated the ventilating bronchoscope which served the purpose of anaesthesia and we could remove the foreign body through its lumen. We were successful in removing the broken tube in 13 small pieces (Figure 2). Check bronchoscopy was clear and the procedure went uneventful.

\section{Case \#2}

A 55-year-old man came to the Emergency Care Unit with the history of sudden onset of difficulty in breathing and cough since 1 day. There was a past history of tracheostomy done two years back for prolonged intubation following head injury. Since two years the patient has been using the same metallic tracheostomy tube on which he was discharged and never came to the hospital for followup. On examination, the patient had tachypnea with reduced air entry on the right side on auscultation and inner flange of the metallic tube was found to be broken below the neck plate. Chest X-ray posterioranterior view revealed the site of the tube in the right main bronchus. Immediately the patient was admitted and taken up for rigid bronchoscopy under general anaesthesia. Of rigid bronchoscope, $6.5 \mathrm{~mm}$ was passed and the broken tube was
Correspondence: Nilam U. Sathe, Department of Ear, Nose and Throat, King Edward Memorial Hospital and Seth G.S. Medical College, Mumbai, India.

Tel: +91.22 .2410 .7000 - Fax: +91.22 .2414 .3435$

E-mail: drneelam_s@yahoo.co.in

Key words: Metallic tracheostomy tube; Broken; Tracheobronchial tree; Foreign body.

Received for publication: 12 February 2016.

Revision received: 20 September 2016.

Accepted for publication: 7 October 2016.

This work is licensed under a Creative Commons Attribution NonCommercial 4.0 License (CC BYNC 4.0).

(C) Copyright N.U. Sathe et al., 2016

Licensee PAGEPress, Italy

Surgical Techniques Development 2016; 6:6466 doi:10.4081/std.2016.6466

found in the right main bronchus and hence removed. Portex tracheostomy tube no. 7.5 was inserted and the post-operative period was uneventful.

\section{Discussion}

Various foreign bodies have been documented in the tracheobronchial tree but fractured tracheostomy tube is quite rare. Cases of broken metallic tube have been reported in the literature being more common with the metallic counterpart. ${ }^{1-5}$ The first instance was reported by Bassoe and Boe in $1960 .{ }^{6}$ Eleven cases of non-synthetic and 12 cases of synthetic tube fracture have been presented by Alvi and Zahtz. ${ }^{7} \mathrm{~A}$ series of nine cases over a period of 8 years has been reported by Gupta in $1996 .{ }^{8}$ Incidence of broken tracheostomy tube that remained undetected for a long period has been rare. Sreenath and Mahendrakar described a case of broken tracheostomy tube that was removed tube four years after inhalation. ${ }^{5}$ Majid concluded that the length of time from first wearing of the tube to its fracture may differ from a few days to eight years. ${ }^{1}$

Metallic tracheostomy tubes are made up of alloy of copper and zinc. ${ }^{9}$ Erosion of the tube may occur due to alkaline reaction by tracheostomy secretions. ${ }^{9,10}$ Breakage is more frequently seen at the junction of the outer shield with the endotracheal part of the tube because there are higher chances of stagnation of secretion at this site where the two parts are welded together. ${ }^{9-11}$ The weak points of the tracheostomy tube are the junctions between the tube and the neck plate, the distal end of the tube and the fenestration 


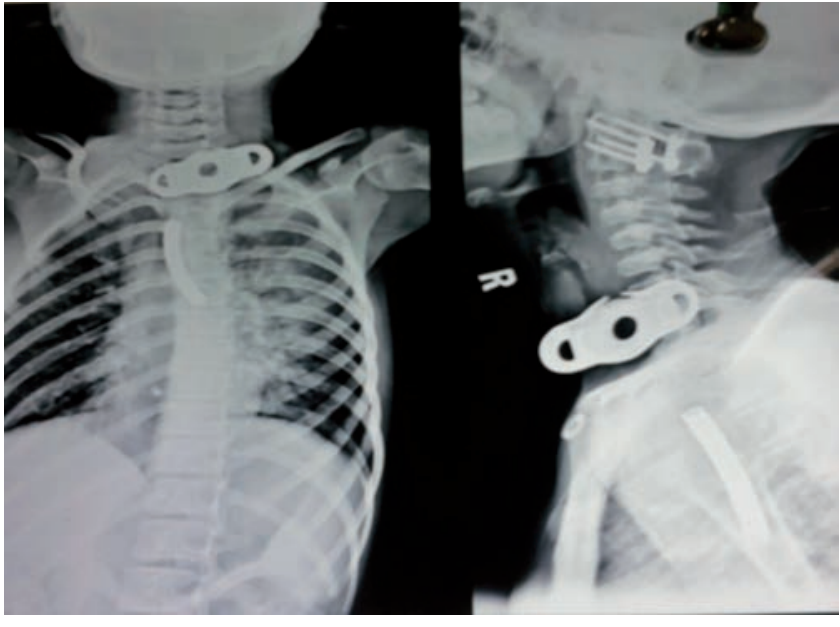

Figure 1. X-ray chest and neck posteroanterior and lateral views showing broken metallic tube in trachea and bronchus.

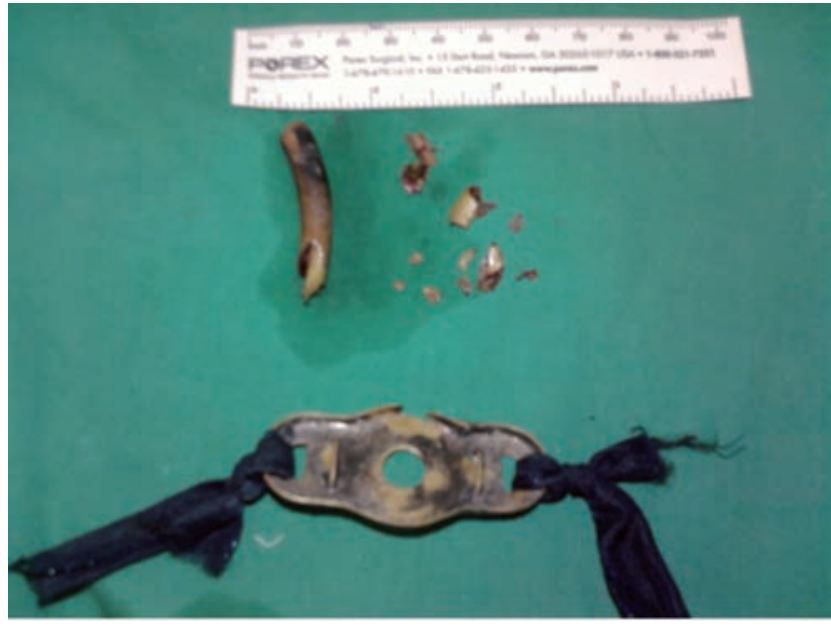

Figure 2. Metallic tube broken into thirteen small pieces. site. ${ }^{1,12,13}$ The most common reported fracture site is at the junction between the tube and the neck plate. Prolonged usage leading to the wear and tear of the tubes have been proposed as the major risk factor for tracheostomy tube fracture. ${ }^{1,12,13}$ The most common dislodged sites reported were the trachea and the right main bronchus. ${ }^{12}$ The predisposing factors leading to fracture of the tracheostomy tube in both our patients included poor followup after tracheostomy and wear and tear as evidenced by the corrosion of the surface of the tube. Such patients mostly present with acute respiratory distress, though there have been cases where the diagnosis have been missed and the tube remained quiescent leading to chronic respiratory distress and patients being wrongly treated for chronic diseases such as pneumonia or chronic bronchitis. ${ }^{1,4,12,13}$

Most of the time it is easily diagnosed on a chest radiograph. ${ }^{4}$ Nowadays, computed tomography of the chest with virtual bronchoscopy may also be helpful in determining the exact site of the broken tube in relation to the tracheobronchial tree in long standing cases, especially when associated chest pathology is suspected. Therapeutic rigid bronchoscopic removal is the mainstay of treatment. The complication can be avoided by proper care and periodic replacement of the worn out tracheostomy tube.

\section{Conclusions}

Hence, we would like to conclude that broken tracheostomy tube presenting as foreign body bronchus is infrequent but it is a preventable complication of tarcheostomy. The patient must be kept on regular followup to check for signs of wear and tear. Timely and periodic replacement of oftracehostomy tube should also be done, otherwise such life-saving surgery can become life-threatening. Patient's education regarding tracheostomy tube is essential in avoiding such complications.

\section{References}

1. Majid AA. Fractured silver tracheostomy tube: a casereport and literature review. Singapore Med J 1989;30:602-4.

2. Rajesh, Bisht SS, Aggarwal V, Bhatia R. Foley's catheter technique for removal of fractured polyvinyl chloride tracheostomy tube in a difficult case. Internet $\mathbf{J}$ Otorhinolarynol 2006;5:1.

3. Wu CT, Lin JJ, Yeh R. Migration of fractured tracheostomy tube into the left main bronchus. Int J Pediatr Otorhi 2007;2:5860 .

4. Gupta SC. Fractured tracheostomy tubes in the tracheobronchial tree: a report of nine cases. J Laryngol Otol 1987;101:861-7.

5. Sreenath J, Mahendrakar V. Management oftracheobronchial foreign bodies: a retrospective analysis. Indian J Otol Head Neck 2002; 54:127-31.

6. Bassoe HH, Boe J. Broken tracheostomy tube as a foreign body. Lancet 1960;1:10067.

7. Alvi A, Zahtz GD. Fracture of synthetic fenestrated tracheostomy tube; case report and review of literature. Am J Otolaryng 1994;15:63-7.

8. Gupta SC, Ahluwalia H. Fractured tracheostomy tube: an overlooked foreign body. J Laryngol Otol 1996;110:1069-71.

9. Kakar PK, Saharaia PS. An unusual forign body in the tracheobronchial tree. J Laryngol Otol 1972;86:1155-7.

10. Maru YK, Puri ND, Majid A. An unusual forign body in the tracheobronchial tree. $\mathrm{J}$ Laryngol Otol 1978;92:1045-8.

11. Ming CC, Ghani SA. Fractured tracheostomy tube in the tracheobronchial tree. $\mathrm{J}$ Laryngol Otol 1989;103:335-6.

12. Piromchai $P$, Lertchanaruengrit $P$, Vatanasapt $\mathrm{P}$, et al. Fractured metallic tracheostomy tube in a child: a case report and review of the literature. J Med Case Rep 2010;4:234.

13. Alqudehy ZA, Alnufaily YK. Fractured tracheostomy tube in the tracheobronchial tree of a child: case report and literature review. J Otol Head Neck 2010;39:70-3. 